\title{
Acute Methylglyoxal-Induced Damage In Blood- Brain Barrier And Hippocampal Tissue
}

\author{
Lílian Juliana Lissner \\ Universidade Federal do Rio Grande do Sul (UFRGS) \\ Krista Minéia Wartchow \\ Universidade Federal do Rio Grande do Sul (UFRGS)

\section{Leticia Rodrigues} \\ Universidade Federal do Rio Grande do Sul (UFRGS)

\section{Larissa Daniele Bobermin} \\ Universidade Federal do Rio Grande do Sul (UFRGS)

\section{Ederson Borba} \\ Universidade Federal do Rio Grande do Sul (UFRGS)

\section{Vitor Gayger Dias} \\ Universidade Federal do Rio Grande do Sul (UFRGS)

\section{Fernanda Hansen} \\ Universidade Federal de Santa Catarina (UFSC)

\section{André Quincozes-Santos} \\ Universidade Federal do Rio Grande do Sul (UFRGS) \\ Carlos-Alberto Gonçalves ( $\sim$ casg@ufrgs.br) \\ Universidade Federal do Rio Grande do Sul (UFRGS)
}

\section{Research Article}

Keywords: astrocyte, integrity, expression

Posted Date: December 10th, 2021

DOI: https://doi.org/10.21203/rs.3.rs-1104792/v1

License: (c) (1) This work is licensed under a Creative Commons Attribution 4.0 International License.

Read Full License 
Acute methylglyoxal-induced damage in blood-brain barrier and hippocampal tissue

Lílian Juliana Lissner ${ }^{\mathrm{a}}$, Krista Minéia Wartchow ${ }^{\mathrm{a}}$, Leticia Rodrigues ${ }^{\mathrm{a}}$, Larissa Daniele Bobermin ${ }^{\mathrm{a}}$, Ederson Borba ${ }^{\mathrm{a}}$, Vitor Gayger Dias ${ }^{\mathrm{a}}$, Fernanda Hansen ${ }^{\mathrm{b}}$, André Quincozes-Santos ${ }^{\mathrm{a}}$, Carlos-Alberto Gonçalves ${ }^{\mathrm{a} *}$

*Corresponding author

${ }^{\text {a }}$ Universidade Federal do Rio Grande do Sul (UFRGS), Biochemistry Graduate Program, Porto Alegre, Brazil

${ }^{\mathrm{b}}$ Universidade Federal de Santa Catarina (UFSC), Department of Nutrition, Nutrition Graduate Program, Florianópolis, Brazil.

*Corresponding author:

Dr Carlos Alberto Gonçalves

Federal University of Rio Grande do Sul (UFRGS), Biochemistry Post-Graduate Program.

Ramiro Barcelos, 2600-Anexo

90035003, Porto Alegre, RS, Brazil

E-mail: casg@ufrgs.br 


\begin{abstract}
Methylglyoxal (MG) is a reactive dicarbonyl compound formed mostly by the glycolytic pathway. Elevated blood glucose levels can cause MG accumulation in plasma and cerebrospinal fluid diabetes mellitus and Alzheimer's disease, where the high reactivity of MG leads to modification of proteins and other biomolecules, generating advanced glycation end products (AGEs) appointed as mediators in those neurodegenerative diseases. Herein, we investigated the blood-brain barrier (BBB) integrity and astrocyte response in the hippocampus to acute insult induced by MG, administered ICV in rats. Seventy-two hours later, a loss of BBB integrity was observed, as assessed by the entry of Evans dye into brain tissue and albumin in the CSF, as well as a decrease of aquaporin-4 and connexin-43 in hippocampal tissue. MG did not induce changes in hippocampal contents of RAGE in this short interval, but decreased the expression of S100B, an astrocyte secreted protein that binds RAGE. The expressions of two important transcription factors of antioxidant response - NfkB and Nrf2, were not changed. However, hemeoxigenase-1 was upregulated in MG-treated group. This data corroborates with the idea that astrocytes, the main cells responsible for MG clearance, are targets of MG toxicity and that BBB dysfunction induced by this compound may contribute to behavioral and cognitive alterations observed in these animals.
\end{abstract}




\section{Introduction}

The blood-brain barrier (BBB) is a complex structure of cells, including endothelial cells, pericytes and astrocytes, which together, regulate the transport of specifics molecules into the brain and limit the entry of blood-derived substances (e.g. immunoglobulins, albumin) (Hawkins and Davis 2005; Engelhardt and Sorokin 2009). Astrocytes contain proteins that are essential to ensure BBB functionality, such as aquaporin 4 (AQP4), which plays a critical role in the cellular water content and flow (Niermann et al. 2001; Badaut et al. 2014), and connexin 43 (CX43), which is a predominant gap junction protein in astrocytes, regulating the passage of ions and metabolites (Lapato and TiwariWoodruff 2018; Xing et al. 2019). Alterations in AQP4 and CX43 expression has been associated with several physiological and pathological conditions (Sáez et al. 2003; Zhang et al. 2015; Verkman et al. 2017; Mader and Brimberg 2019).

Methylglyoxal (MG) is a reactive dicarbonyl compound formed mainly by glycolytic pathway (Thornalley 1993; Angeloni et al. 2014). The chronic elevation of blood glucose results in MG accumulation in plasma and cerebrospinal levels that are encountered in patients with diabetes mellitus (DM) and Alzheimer's disease (AD) (Thornalley 1993; Kuhla et al. 2005). The high reactivity of MG leads to modification of proteins and other biomolecules, generating advanced glycated end products (AGEs) (Ahmed and Thornalley 2003; Rabbani and Thornalley 2008). Studies with diabetes models in rodents have shown that MG induces a brain endothelial cells dysfunction through barrier-protein glycation (Li et al. 2015), impairing the BBB function and integrity (Beeri 2011; Rabbani and Thornalley 2015; Hussain et al. 2016), triggering inflammatory response, incrementing permeability, causing cellular morphology changes (Tóth et al. 2014b; Hussain et al. 2016) and disrupting gap junctions (Li 2013; Tóth et al. 2014a).

The cellular accumulation of AGEs alters the protein machinery including nuclear factors (Pugliese 2008) and contributes to abnormal formation of proteins cross-linking, which causes the functional changes typical of chronic diabetic complications (Sell et al. 1992). The activation of receptor for of AGEs (RAGE) leads to cellular signaling involving many pathways, including the nuclear factor $\mathrm{kB}(\mathrm{NfkB})$, mainly associated to inflammatory response (Piarulli et al. 2013; Saleh et al. 2019). Together with NfkB, the nuclear factor erythroid-2 related factor (Nrf2), regulates the inflammatory response, beyond the modulation of metabolic oxidative response (Bellezza et al. 2018). Nrf2 is a protein involved in the restoring of intracellular balance between oxidants and antioxidants after oxidative insult and regulation of numerous genes and enzymes (e.g heme-oxigenase 1 (HO-1) (Minelli et al. 2009; Jian et al. 2011; Zhang et al. 2013; Choi et al. 2016; Wang 2019). Nrf2 deletion may contribute to the increased sensitivity to oxidative stress and inflammation (Jakel et al. 2007; Ichihara et al. 2010; Taki-Nakano et al. 2014; Zhao et al. 2019). It has been demonstrated that Nrf2 mediates a signaling pathway against MG toxicity (de Oliveira et al. 2015) as well as prevents the damage caused by high concentrations of glucose in animal models of diabetes (Choi et al. 2016; Zhao et al. 2019).

There are evidence that chronic elevated levels of MG alters the BBB and consequently contribute to brain changes observed in DM and neurodegenerative diseases (Fang et al. 2015, p. 201; Hussain et al. 2016; Kim et al. 2020). However, few studies address the acute effect of MG, particularly in vivo on barrier integrity and neuroglial functions. In previous studies of our group, we observed that acute intracerebroventricular (ICV) administration of elevated concentrations of MG impairs short-and long-term learning and memory processes and glial function (Lissner et al. 2021). Seeking for a better understanding of this previous findings, herein, our focus was to investigate how high concentration of MG in vivo affects the BBB function and integrity and if this fact could modulate inflammatory pathways and cellular stress responses in astrocytes from hippocampus, which is a sensible brain area affected in neurodegenerative diseases. 


\subsection{Reagents}

Methylglyoxal (MG) solution (40\% in $\mathrm{H}_{2} \mathrm{O}$ ), Triton X-100, o-phthaldialdehyde, S100B protein, anti-S100B (SHB1) were purchased from Sigma-Aldrich (Saint Louis, MO, USA). Polyclonal anti-S100B was purchased from DAKO (São Paulo, SP, Brazil) and Evans blue Dye (EBD) (Neon Comercial, São Paulo, Brazil). Immunoblots were performed using the following antibodies: Rabbit polyclonal (anti-AQP4 and anti-Cx43), mouse-monoclonal (anti- RAGE), purchased form Millipore, Darmstadt, Germany and HRP-conjugated $\beta$-actin antibody (Proteintech, Rosemont, IL, USA). Peroxidase secondaries antibodies from GE (Little Chalfont, United Kingdom). All others chemicals were purchased from local commercial suppliers.

\subsection{Animals}

Male Wistar rats (90-days old) were obtained from our breeding colony (Department of Biochemistry, UFRGS, Porto Alegre, Brazil). The rats were maintained under controlled light and environmental conditions (12 h light/12 h dark cycle at a constant temperature of $22 \pm 1^{\circ} \mathrm{C}$ ) and had free access to commercial chow and water.

\subsection{Surgical procedure}

MG was unilaterally infused in the lateral ventricle as described in previous studies (Rodrigues et al. 2019; Lissner et al. 2021). The rats were divided in two groups: SHAM operated and MG-treated. Briefly, rats were anesthetized with ketamine/xylazine (75 and $10 \mathrm{mg} / \mathrm{kg}$, respectively, i.p) and positioned in stereotaxic apparatus. A midline sagittal incision was made in the scalp and burr holes were drilled in the skull on right sides over the lateral ventricle. The right ventricle was accessed using the following coordinates: $0.9 \mathrm{~mm}$ posterior to the bregma; $1.5 \mathrm{~mm}$ lateral to the sagittal suture; $3.6 \mathrm{~mm}$ beneath the surface of the brain. Animals received $5 \mu \mathrm{L}$ of MG ICV unilaterally with a final concentration of $3 \mu \mathrm{mol} / \mu \mathrm{L}$; the SHAM group received an equal volume of Hank's balanced salt solution - HBSS, pH 7.2. After the surgical procedure, animals were kept warm until recovery from anesthesia. Approximately seventy-two hours after MG injection, rats were euthanized and biochemical parameters were evaluated.

\subsection{Biochemical analysis}

\subsubsection{Obtaining cerebrospinal fluid, serum and hippocampal slices}

Animals were anesthetized and then positioned in a stereotaxic holder for CSF collection of $100 \mu \mathrm{L}$ (approximately) from the cisterna magna. The puncture was performed using an insulin syringe (27gauge x 1/2" length). Rats were then removed from the stereotaxic apparatus and placed in a flat place; whole blood was obtained through an intracardiac puncture using a $0.37-\mathrm{mm}$ diameter needle and was collected into clot activator tubes, centrifuged (3000 rpm, $10 \mathrm{~min}, 4^{\circ} \mathrm{C}$ ) to separate serum. The hippocampi were dissected and transverse of $0.3 \mathrm{~mm}$ were obtained using a McElwain Tissue Chopper. The samples were frozen $-80^{\circ} \mathrm{C}$ until posterior analysis (Netto et al. 2006).

\subsubsection{Determination of CSF/Serum Albumin Ratio}

The CSF/serum albumin ratio was analyzed using the bromocresol green assay kit from Doles (Goiânia, Brazil) as described to (Durgawale et al. 2005), modified. $10 \mu \mathrm{L}$ of serum or $50 \mu \mathrm{L}$ of CSF was mixture with $2 \mathrm{~mL}$ of color 
reagent. The samples were incubated for $10 \mathrm{~min}$ at $37^{\circ} \mathrm{C}$, and the absorbance was measured at $546 \mathrm{~nm}$ against a reagent blank.

\subsubsection{In vivo EBD injection and dye extraction}

BBB permeability was evaluated according to the distribution in the rat brain of EBD administered at a dose of $2 \mathrm{mg} / \mathrm{kg}$ by an intracarotid injection (Manaenko et al. 2011; Wang and Lai 2015), modified. After a two-hours period to enable uniform EBD distribution, the rats were anesthetized using ketamine/xylazine and were perfused through the left cardiac ventricle with $200 \mathrm{~mL}$ of saline solution. The brains were removed, weighed and the content of the dye extracted from each brain was determined with a spectrophotometer (at $620 \mathrm{~nm}$ ). The quantitative calculation of the dye content was based on (Wang and Lai 2015).

\subsubsection{Western blot analysis}

Proteins from hippocampal sample were homogenized in a sample buffer [62.5 mM Tris- $\mathrm{HCl} \mathrm{pH} 6.8,2 \%(\mathrm{w} / \mathrm{v})$ SDS, $5 \%$ (w/v) $\beta$-mercaptoethanol, $10 \%$ (v/v) glycerol, $0.002 \%$ (w/v) bromophenol blue] and separated by SDS-PAGE on $12 \%$ sodium dodecyl sulfate polyacrylamide and electro transferred onto nitrocellulose membranes. Membranes were blocked overnight at $4{ }^{\circ} \mathrm{C}$ with $2 \%$ chicken egg albumin in Tris-buffered saline with Tween 20 (TTBS). After incubation overnight at $4^{\circ} \mathrm{C}$ with primary antibodies (anti-AQP4, anti-Cx43 and anti-RAGE at dilution 1:5000), the membranes were incubated for $1 \mathrm{~h}$ at room temperature and exposed to horseradish peroxidase-linked anti-IgG antibodies. Chemiluminescent bands were detected by image analyzer (Image Quant LAS4000 from GE) and optical density was quantified using ImageJ software. The results were expressed as percentages of SHAM.

\subsubsection{RNA extraction and quantitative RT-PCR}

Total RNA was isolated from hippocampal slices using TRIzol Reagent (Invitrogen, Carlsbad, CA). The concentration and purity of the RNA were determined spectrophotometrically at a ratio of 260:280. $1 \mu \mathrm{g}$ of extracted RNA was reverse transcribed using High-Capacity cDNA Reverse Transcription Kit (Applied Biosystems, Thermo Fisher Scientific) in a $20 \mu \mathrm{L}$ reaction according to manufacturer's instructions. The messenger RNA (mRNA) encoding HMGB1 (\#Rn02377062_g1), HO-1 (\#Rn01536933_m1), Nrf2 (\#Rn00582415_m1) and p50 NfkB (\#Rn01399572_m1) were quantified using the TaqMan real-time RT-PCR system using inventory primers and probes purchased from Applied Biosystems (Bobermin et al. 2019). Target mRNA levels were normalized to $\beta$-actin (\#Rn00667869_m1) levels. Results were analyzed employing the $2^{-\Delta \Delta \mathrm{Ct}}$ method (Bobermin et al. 2020, p. 20) adapted from (Livak and Schmittgen 2001) and expressed relative to the levels of SHAM group.

\subsection{Protein determination}

Protein content was measured by Lowry's method using bovine serum albumin as standard (Peterson 1977).

\subsection{Statistical analysis}

Data are reported as means \pm standard errors and analyzed statistically by Student's $t$-test. Differences were considered significant when $\mathrm{p} \leq 0.05$. All analyses were performed using the Prism 6.0 (GraphPad).

\section{Results}

For all techniques performed, the samples were obtained seventy-hours after ICV administration of MG. 


\subsection{MG infusion disrupted BBB permeability and increased albumin levels}

Initially we evaluated the effect of MG-ICV on functional permeability of BBB using the Evans blue staining assay and the CSF/serum albumin ratio. The concentration of EBD extracted of total brain was increased in the MG group (Fig. 1A, $\mathrm{p}=0.001$ ) and concomitantly, the CSF/serum albumin ratio was increased drastically in MG-treated group when compared with SHAM group (Fig. 1B, p<0.0001).

\subsection{Decreased of AQP4 and Cx43 levels in MG-treated group}

To identify alterations in astroglial connectivity related to BBB, we measured the levels of proteins AQP4 and Cx43 by western blotting. Here, it was observed a decrease of AQP4 (Fig. 2A, p = 0.04) and CX43 (Fig. 2B, p = 0.01) levels.

\subsection{RAGE signaling is altered in MG-treated group}

In order to understand if RAGE signaling is involved, we measured the contents of RAGE and S100B, a RAGE ligand secreted by astrocytes. No changes were observed in RAGE (Fig 3A, p =0.89) or S100B (Fig 3B, p = 0.19). Then we evaluated mRNA levels of S100B and HMGB1, another RAGE ligand. Interestingly, in MG-treated we observed an up-regulation of mRNA levels for S100B (Fig 3C, p =0.04), but not for HMGB1 (Fig 3D, $p=0.16$ ).

\subsection{MG exposure increased HO-1 mRNA expression in the hippocampus}

Then, we investigated hippocampal inflammatory pathways possibly affected by MG exposure. No changes were observed in mRNA levels for NfkB (Fig 4A, p =0.74) and Nfr2 (Fig 4B, p = 0.82) in hippocampal tissue of animals exposed to MG. However, it was observed an increase of mRNA levels for HO-1 in the MG-group (Fig. 4C, p = 0.03).

\section{Discussion}

The carbonyl stress is triggered by accumulation of reactive species as MG (Ramasamy et al. 2006) and the risk of glycation levels in cardiovascular and cerebrovascular system of diabetic and Alzheimer's diseases patients are elevated (Lapolla et al. 2003; Vander Jagt and Hunsaker 2003; Tóth et al. 2014a). Previous studies demonstrated that, in hyperglycemic conditions, MG induces endothelial barrier microvascular dysfunction (Li 2013; Hussain et al. 2016; Irshad et al. 2019), a reduced integrity and increased BBB permeability (Tóth et al. 2014b, a) and contributes to a higher progression of vascular complications and cerebrovascular injury (Fang et al. 2015). Vascular alterations in diabetes are linked with breakdown of BBB functioning (Hawkins et al. 2007) and these alterations contribute functional and structurally with brain changes and cognitive impairment observed in patients and experimental models (Serlin et al. 2011; Zanotto et al. 2017). We had previously showed that the acute insult with MG affects astroglial activity (Lissner et al. 2021) and considering the involvement of these cells in BBB, we decided to evaluate this possibility more closely.

MG per se and/or AGEs induce oxidative stress and inflammatory pathways activation through interaction with RAGE (Kalapos 2008; Ramasamy et al. 2011; Liu et al. 2013; Choi et al. 2016; MacLean et al. 2019). Exacerbated expression of RAGE has been implicated in many neurodegenerative disorders, which can be activated by AGEs as well as the ligands HMGB1 and S100B (Sagheddu et al. 2018; MacLean et al. 2019).

In this acute model of brain lesion, elevated extracellular levels S100B were observed in MG-treated animals. On the other hand, mRNA levels for S100B (but not HMGB1) are reduced in the hippocampus, confirming an astroglia commitment due to MG exposure. At this time, we can think but do not know for certainty if this reduction in S100B gene expression is related to the reduction of protein secretion in hippocampal slices exposed directly to MG (Hansen et 
al. 2016a). Here, we not observed difference in the hippocampal immune contents of RAGE. These data corroborate with a previous study from our group (Hansen et al. 2016a), which suggests a non-involvement of RAGE when hippocampal slices were treated with high concentration of MG. In agreement, our suggestion is that more studies are necessary to confirm the idea that MG could triggers an inflammatory process via non-receptor pathway or other glial or neuronal AGE receptors (Vlassara 2001; Ott et al. 2014). Considering such changes, therefore, it is possible to conceive an alteration in the S100B / RAGE communication in this acute model, which could be occurring in situations of hyperglycemic peaks.

Considering the importance of understanding more about the regulation of astrocytic inflammatory response in this model, we also investigated the levels of two main transcription factors, possibly activated - NfkB and Nfr2, as well as the homeoxygenase - 1 (HO-1), an enzyme related to neuroprotection (Nitti et al. 2018). Activation of Nfr2 has been associated with modulation of antioxidant and detoxifying enzymes expression, including HO-1 (Syapin 2009; Calkins et al. 2009; Niture et al. 2014) and its absence is related to cellular damage by increase of oxidative stress molecules and inflammation (Ichihara et al. 2010; Taki-Nakano et al. 2014). Oxidative stress environment can also activate NFkB and concomitantly releasing of proinflammatory cytokines (Patel and Santani 2009).

We observed no changes in the expression of Nrf2 and NfkB under acute MG exposure. However, the mRNA levels for HO-1 were increased. These data suggest that this up-regulation of HO-1 would be a protection response against the acute insult generated by MG. These data confirm the importance of HO-1 against MG toxicity and point out that other transcription factors, in addition to $\mathrm{Nfr} 2$ and $\mathrm{NfkB}$, must be involved in the most initial antioxidant response (Nitti et al. 2018).

In addition, we observed an acute BBB rupture measured by a higher EBD concentration in brain tissue and an increased CSF/serum albumin ratio in MG group. It is known that long-term incubation with high MG concentration leads to a dramatic change in the permeability of BBB endothelial cells, possibly due to glycation of tight junction proteins (Hussain et al. 2016). It has been showed that MG binds to specific basic sites of proteins causing endothelial cell detachment in brain tissue (McDonald et al. 2009). Although we have not characterized the glycated proteins of BBB in this acute MG-induced model of damage, it is clear the functional impairment in BBB, evidenced by the greater permeability of serum albumin and Evans blue dye.

A comment needs to be made here. In the previous work we showed that levels of S100B are increased in the CSF but not in the serum of animals submitted to ICV administration of MG (Lissner et al. 2021). Therefore, even if there is a clear loss of the integrity of the BBB, the S100B does not flow from the CSF to the serum in this acute model. This is especially important considering that the serum increase in S100B by many authors is attributed to the loss of BBB integrity and these data contradict this idea.

The BBB disruption may affect the balance of water and ion homeostasis as well as impair the intercellular communication in astrocytic cells (Chu 2016; Hussain et al. 2016). Astroglial impairment induced by MG has been described in cell cultures (Chu 2016; Hansen et al. 2017) and hippocampal slices directly exposed (Hansen et al. 2016a) and hippocampal slices after ICV administration (Hansen et al. 2016b). Considering the importance of astrocytes to BBB function, we investigated whether glial parameters related to the activity of this barrier are altered in this model of acute injury produced by MG.

Our results showed that the MG-treated group exhibited a decrease in the hippocampal AQP4 and Cx43 levels. Cx43 is an important connexin for BBB functionality because it is involved in the gap communication between endothelial cells and also between astrocytes (Kovács et al. 2012; Johnson et al. 2018). AGE-albumin treatment of aortic endothelial 
cells reduced gap communications (Wang et al. 2011). Our data suggests that the impairment of gap communication dependent on Cx43 caused by MG exposure affects BBB functionality and astroglial syncytium in the hippocampus.

Moreover, we found a decrease of hippocampal AQP4. This water channel is highly expressed in astrocytes, including in the endfoots surrounding endothelium in the BBB. Although we do not know whether these proteins are direct targets of MG-mediated glycation, these data show, for the first time, the acute impairment of BBB cell connectivity to this glucose derivative. Changes in CX43 and AQP4 reveal an acute failure in BBB connectivity due to MG exposure, which could compromise aqueous and ionic homeostasis.

It is important to mention some limitations of this study. Firstly, the MG concentration under pathological conditions (e.g., diabetes), is lower than that used in this study. However, this concentration was based on previous studies of ICV administration, and it seemed appropriate to study an acute MG insult in BBB. Secondly, euthanasia was performed seventy hours after the administration of MG and shorter times would allow a better temporal correlation between functional and neurochemical changes. However, in 72 hours it is possible to disregard the free circulating presence of MG since the endogenous metabolism by glyoxalase system is approximately 10 minutes. Finally, we used a commercial MG and did not analyze the purity of this compound. We cannot exclude the possibility that impurities may have contributed to the observed effects.

\section{Conclusions}

Our findings demonstrated that elevated levels of MG, here administered by ICV infusion, were able to induce loss of BBB integrity in short time, measured by increment of permeability and decrease of AQP4 and Cx43 levels. This reinforce the idea that astrocytes, the main cells responsible for MG clearance, are targets of this compound and that BBB dysfunction may contribute to behavioral and cognitive alterations observed in these animals. The decrease of mRNA for S100B emphasizes the glial susceptibility to damage caused by MG. Finally, the defense against the acute MG-induced oxidative stress involves an upregulation of HO-1, which initial expression does not seem concomitantly involve Nfr2 and/or NfkB expression. 
Ahmed N, Thornalley PJ (2003) Quantitative screening of protein biomarkers of early glycation, advanced glycation, oxidation and nitrosation in cellular and extracellular proteins by tandem mass spectrometry multiple reaction monitoring. Biochemical Society Transactions 31:6

Angeloni C, Zambonin L, Hrelia S (2014) Role of Methylglyoxal in Alzheimer's Disease. BioMed Research International 2014:1-12. https://doi.org/10.1155/2014/238485

Badaut J, Fukuda AM, Jullienne A, Petry KG (2014) Aquaporin and brain diseases. Biochimica et Biophysica Acta (BBA) - General Subjects 1840:1554-1565. https://doi.org/10.1016/j.bbagen.2013.10.032

Beeri MS (2011) Serum concentration of an inflammatory glycotoxin, methylglyoxal, is associated with increased cognitive decline in elderly individuals. Mechanisms of Ageing and Development 5

Bellezza I, Giambanco I, Minelli A, Donato R (2018) Nrf2-Keap1 signaling in oxidative and reductive stress. Biochimica et Biophysica Acta (BBA) - Molecular Cell Research 1865:721-733. https://doi.org/10.1016/j.bbamcr.2018.02.010

Bobermin LD, Quincozes-Santos A, Santos CL, et al (2020) Zika virus exposure affects neuron-glia communication in the hippocampal slices of adult rats. Sci Rep 10:21604. https://doi.org/10.1038/s41598-020-78735-y

Bobermin LD, Roppa RHA, Quincozes-Santos A (2019) Adenosine receptors as a new target for resveratrol-mediated glioprotection. Biochimica et Biophysica Acta (BBA) - Molecular Basis of Disease 1865:634-647. https://doi.org/10.1016/j.bbadis.2019.01.004

Calkins MJ, Johnson DA, Townsend JA, et al (2009) The Nrf2/ARE Pathway as a Potential Therapeutic Target in Neurodegenerative Disease. Antioxidants \& Redox Signaling 11:497-508. https://doi.org/10.1089/ars.2008.2242

Choi EM, Suh KS, Kim YJ, et al (2016) Glabridin Alleviates the Toxic Effects of Methylglyoxal on Osteoblastic MC3T3E1 Cells by Increasing Expression of the Glyoxalase System and Nrf2/HO-1 Signaling and Protecting Mitochondrial Function. J Agric Food Chem 64:226-235. https://doi.org/10.1021/acs.jafc.5b05157

Chu JMT (2016) Methylglyoxal-induced neuroinflammatory response in in vitro astrocytic cultures and hippocampus of experimental animals. Metab Brain Dis 10

de Oliveira MR, Ferreira GC, Schuck PF, Dal Bosco SM (2015) Role for the PI3K/Akt/Nrf2 signaling pathway in the protective effects of carnosic acid against methylglyoxal-induced neurotoxicity in SH-SY5Y neuroblastoma cells. Chemico-Biological Interactions 242:396-406. https://doi.org/10.1016/j.cbi.2015.11.003

Durgawale P, Kanase S, Shukla PS, Sontakke S (2005) A sensitive and economical modified method for estimation of cerebrospinal fluid proteins. Indian Journal of Clinical Biochemistry 20:174-177. https://doi.org/10.1007/BF02867422

Engelhardt B, Sorokin L (2009) The blood-brain and the blood-cerebrospinal fluid barriers: function and dysfunction. Seminars in Immunopathology 31:497-511. https://doi.org/10.1007/s00281-009-0177-0

Fang L, Li X, Zhong Y, et al (2015) Autophagy protects human brain microvascular endothelial cells against methylglyoxal-induced injuries, reproducible in a cerebral ischemic model in diabetic rats. Journal of Neurochemistry 135:431-440. https://doi.org/10.1111/jnc.13277

Hansen F, Battú CE, Dutra MF, et al (2016a) Methylglyoxal and carboxyethyllysine reduce glutamate uptake and S100B secretion in the hippocampus independently of RAGE activation. Amino Acids 48:375-385. https://doi.org/10.1007/s00726-015-2091-1

Hansen F, Galland F, Lirio F, et al (2017) Methylglyoxal Induces Changes in the Glyoxalase System and Impairs Glutamate Uptake Activity in Primary Astrocytes. Oxidative Medicine and Cellular Longevity 2017:1-11. https://doi.org/10.1155/2017/9574201 
Hansen F, Pandolfo P, Galland F, et al (2016b) Methylglyoxal can mediate behavioral and neurochemical alterations in rat brain. Physiology \& Behavior 164:93-101. https://doi.org/10.1016/j.physbeh.2016.05.046

Hawkins BT, Davis TP (2005) The Blood-Brain Barrier/Neurovascular Unit in Health and Disease. Pharmacol Rev 57:173-185. https://doi.org/10.1124/pr.57.2.4

Hawkins BT, Lundeen TF, Norwood KM, et al (2007) Increased blood-brain barrier permeability and altered tight junctions in experimental diabetes in the rat: contribution of hyperglycaemia and matrix metalloproteinases. Diabetologia 50:202-211. https://doi.org/10.1007/s00125-006-0485-z

Hussain M, Bork K, Gnanapragassam VS, et al (2016) Novel insights in the dysfunction of human blood-brain barrier after glycation. Mechanisms of Ageing and Development 155:48-54. https://doi.org/10.1016/j.mad.2016.03.004

Ichihara S, Yamada Y, Liu F, et al (2010) Ablation of the Transcription Factor Nrf2 Promotes Ischemia-Induced Neovascularization by Enhancing the Inflammatory Response. ATVB 30:1553-1561. https://doi.org/10.1161/ATVBAHA.110.204123

Irshad Z, Xue M, Ashour A, et al (2019) Activation of the unfolded protein response in high glucose treated endothelial cells is mediated by methylglyoxal. Scientific Reports 9:7889. https://doi.org/10.1038/s41598-019-44358-1

Jakel RJ, Townsend JA, Kraft AD, Johnson JA (2007) Nrf2-mediated protection against 6-hydroxydopamine. Brain Res 1144:192-201. https://doi.org/10.1016/j.brainres.2007.01.131

Jian Z, Li K, Liu L, et al (2011) Heme Oxygenase-1 Protects Human Melanocytes from H2O2-Induced Oxidative Stress via the Nrf2-ARE Pathway. Journal of Investigative Dermatology 131:1420-1427. https://doi.org/10.1038/jid.2011.56

Johnson AM, Roach JP, Hu A, et al (2018) Connexin 43 gap junctions contribute to brain endothelial barrier hyperpermeability in familial cerebral cavernous malformations type III by modulating tight junction structure. The FASEB Journal 32:2615-2629. https://doi.org/10.1096/fj.201700699R

Kalapos MP (2008) The tandem of free radicals and methylglyoxal. Chem Biol Interact 171:251-271. https://doi.org/10.1016/j.cbi.2007.11.009

Kim D, Kim K-A, Kim J-H, et al (2020) Methylglyoxal-Induced Dysfunction in Brain Endothelial Cells via the Suppression of Akt/HIF-1 $\alpha$ Pathway and Activation of Mitophagy Associated with Increased Reactive Oxygen Species. Antioxidants (Basel) 9:. https://doi.org/10.3390/antiox9090820

Kovács R, Heinemann U, Steinhäuser C (2012) Mechanisms underlying blood-brain barrier dysfunction in brain pathology and epileptogenesis: role of astroglia. Epilepsia 53 Suppl 6:53-59. https://doi.org/10.1111/j.15281167.2012.03703.x

Kuhla B, Lüth H-J, Haferburg D, et al (2005) Methylglyoxal, Glyoxal, and Their Detoxification in Alzheimer's Disease. Annals of the New York Academy of Sciences 1043:211-216. https://doi.org/10.1196/annals.1333.026

Lapato AS, Tiwari-Woodruff SK (2018) Connexins and pannexins: At the junction of neuro-glial homeostasis \& disease. J Neuro Res 96:31-44. https://doi.org/10.1002/jnr.24088

Lapolla A, Flamini R, Vedova AD, et al (2003) Glyoxal and Methylglyoxal Levels in Diabetic Patients: Quantitative Determination by a New GC/MS Method. Clinical Chemistry and Laboratory Medicine 41:. https://doi.org/10.1515/CCLM.2003.180

Li W (2013) Acute carbonyl stress induces occludin glycation and brain microvascular endothelial barrier dysfunction Role for glutathione-dependent metabolism of methylglyoxal. Free Radical Biology and Medicine 11

Li W, Maloney RE, Aw TY (2015) High glucose, glucose fluctuation and carbonyl stress enhance brain microvascular endothelial barrier dysfunction: Implications for diabetic cerebral microvasculature. Redox Biology 5:80-90. https://doi.org/10.1016/j.redox.2015.03.005 
Lissner LJ, Rodrigues L, Wartchow KM, et al (2021) Short-Term Alterations in Behavior and Astroglial Function After Intracerebroventricular Infusion of Methylglyoxal in Rats. Neurochem Res 46:183-196. https://doi.org/10.1007/s11064-020-03154-4

Liu Y-W, Zhu X, Yang Q-Q, et al (2013) Suppression of methylglyoxal hyperactivity by mangiferin can prevent diabetesassociated cognitive decline in rats. Psychopharmacology 228:585-594. https://doi.org/10.1007/s00213-013$3061-5$

Livak KJ, Schmittgen TD (2001) Analysis of Relative Gene Expression Data Using Real-Time Quantitative PCR and the 2- $\Delta \Delta$ CT Method. Methods 25:402-408. https://doi.org/10.1006/meth.2001.1262

MacLean M, Derk J, Ruiz HH, et al (2019) The Receptor for Advanced Glycation End Products (RAGE) and DIAPH1: Implications for vascular and neuroinflammatory dysfunction in disorders of the central nervous system. Neurochem Int 126:154-164. https://doi.org/10.1016/j.neuint.2019.03.012

Mader S, Brimberg L (2019) Aquaporin-4 Water Channel in the Brain and Its Implication for Health and Disease. Cells 8:90. https://doi.org/10.3390/cells 8020090

Manaenko A, Chen H, Kammer J, et al (2011) Comparison Evans Blue injection routes: Intravenous versus intraperitoneal, for measurement of blood-brain barrier in a mice hemorrhage model. J Neurosci Methods 195:206-210. https://doi.org/10.1016/j.jneumeth.2010.12.013

McDonald DM, Coleman G, Bhatwadekar A, et al (2009) Advanced glycation of the Arg-Gly-Asp (RGD) tripeptide motif modulates retinal microvascular endothelial cell dysfunction. Mol Vis 15:1509-1520

Minelli A, Conte C, Grottelli S, et al (2009) Cyclo(His-Pro) up-regulates heme oxygenase 1 via activation of Nrf2-ARE signalling. Journal of Neurochemistry 111:956-966. https://doi.org/10.1111/j.1471-4159.2009.06376.x

Netto CBO, Conte S, Leite MC, et al (2006) Serum S100B Protein Is Increased in Fasting Rats. Archives of Medical Research 37:683-686. https://doi.org/10.1016/j.arcmed.2005.11.005

Niermann H, Amiry-Moghaddam M, Holthoff K, et al (2001) A Novel Role of Vasopressin in the Brain: Modulation of Activity-Dependent Water Flux in the Neocortex. J Neurosci 21:3045-3051. https://doi.org/10.1523/JNEUROSCI.21-09-03045.2001

Nitti M, Piras S, Brondolo L, et al (2018) Heme Oxygenase 1 in the Nervous System: Does It Favor Neuronal Cell Survival or Induce Neurodegeneration? Int J Mol Sci 19:. https://doi.org/10.3390/ijms19082260

Niture SK, Khatri R, Jaiswal AK (2014) Regulation of Nrf2 — an update. Free Radical Biology and Medicine 66:36-44. https://doi.org/10.1016/j.freeradbiomed.2013.02.008

Ott C, Jacobs K, Haucke E, et al (2014) Role of advanced glycation end products in cellular signaling. Redox Biol 2:411429. https://doi.org/10.1016/j.redox.2013.12.016

Patel S, Santani D (2009) Role of NF-kB in the pathogenesis of diabetes and its associated complications. Pharmacological Reports 9

Peterson GL (1977) A simplification of the protein assay method of Lowry et al. which is more generally applicable. Analytical Biochemistry 83:346-356. https://doi.org/10.1016/0003-2697(77)90043-4

Piarulli F, Sartore G, Lapolla A (2013) Glyco-oxidation and cardiovascular complications in type 2 diabetes: a clinical update. Acta Diabetol 50:101-110. https://doi.org/10.1007/s00592-012-0412-3

Pugliese G (2008) Do advanced glycation end products contribute to the development of long-term diabetic complications? Nutrition, Metabolism and Cardiovascular Diseases 18:457-460. https://doi.org/10.1016/j.numecd.2008.06.006

Rabbani N, Thornalley PJ (2015) Dicarbonyl stress in cell and tissue dysfunction contributing to ageing and disease. Biochemical and Biophysical Research Communications 458:221-226. https://doi.org/10.1016/j.bbrc.2015.01.140 
Rabbani N, Thornalley PJ (2008) Dicarbonyls linked to damage in the powerhouse: glycation of mitochondrial proteins and oxidative stress. Biochm Soc Trans 36:1045-1050. https://doi.org/10.1042/BST0361045

Ramasamy R, Yan SF, Schmidt AM (2006) Methylglyoxal Comes of AGE. Cell 124:258-260. https://doi.org/10.1016/j.cell.2006.01.002

Ramasamy R, Yan SF, Schmidt AM (2011) Receptor for AGE (RAGE): signaling mechanisms in the pathogenesis of diabetes and its complications: RAGE, signal transduction, and diabetes. Annals of the New York Academy of Sciences 1243:88-102. https://doi.org/10.1111/j.1749-6632.2011.06320.x

Rodrigues L, Wartchow KM, Suardi LZ, et al (2019) Streptozotocin causes acute responses on hippocampal S100B and BDNF proteins linked to glucose metabolism alterations. Neurochem Int 128:85-93. https://doi.org/10.1016/j.neuint.2019.04.013

Sáez JC, Berthoud VM, Brañes MC, et al (2003) Plasma Membrane Channels Formed by Connexins: Their Regulation and Functions. Physiological Reviews 83:1359-1400. https://doi.org/10.1152/physrev.00007.2003

Sagheddu R, Chiappalupi S, Salvadori L, et al (2018) Targeting RAGE as a potential therapeutic approach to Duchenne muscular dystrophy. Hum Mol Genet 27:3734-3746. https://doi.org/10.1093/hmg/ddy288

Saleh I, Maritska Z, Parisa N, Hidayat R (2019) Inhibition of Receptor for Advanced Glycation End Products as New Promising Strategy Treatment in Diabetic Retinopathy. OAMJMS 7:. https://doi.org/10.3889/oamjms.2019.759

Sell DR, Lapolla A, Odetti P, et al (1992) Pentosidine formation in skin correlates with severity of complications in individuals with long-standing IDDM. Diabetes 41:1286-1292. https://doi.org/10.2337/diab.41.10.1286

Serlin Y, Levy J, Shalev H (2011) Vascular pathology and blood-brain barrier disruption in cognitive and psychiatric complications of type 2 diabetes mellitus. Cardiovasc Psychiatry Neurol 2011:609202. https://doi.org/10.1155/2011/609202

Syapin PJ (2009) Regulation of haeme oxygenase-1 for treatment of neuroinflammation and brain disorders: Regulating HO-1 in the CNS. British Journal of Pharmacology 155:623-640. https://doi.org/10.1038/bjp.2008.342

Taki-Nakano N, Ohzeki H, Kotera J, Ohta H (2014) Cytoprotective effects of 12-oxo phytodienoic acid, a plant-derived oxylipin jasmonate, on oxidative stress-induced toxicity in human neuroblastoma SH-SY5Y cells. Biochimica et Biophysica Acta (BBA) - General Subjects 1840:3413-3422. https://doi.org/10.1016/j.bbagen.2014.09.003

Thornalley PJ (1993) The glyoxalase system in health and disease. Molecular Aspects of Medicine 14:287-371. https://doi.org/10.1016/0098-2997(93)90002-U

Tóth AE, Tóth A, Walter FR, et al (2014a) Compounds Blocking Methylglyoxal-induced Protein Modification and Brain Endothelial Injury. Archives of Medical Research 45:753-764. https://doi.org/10.1016/j.arcmed.2014.10.009

Tóth AE, Walter FR, Bocsik A, et al (2014b) Edaravone Protects against Methylglyoxal-Induced Barrier Damage in Human Brain Endothelial Cells. PLoS ONE 9:e100152. https://doi.org/10.1371/journal.pone.0100152

Vander Jagt DL, Hunsaker LA (2003) Methylglyoxal metabolism and diabetic complications: roles of aldose reductase, glyoxalase-I, betaine aldehyde dehydrogenase and 2-oxoaldehyde dehydrogenase. Chem Biol Interact 143144:341-351. https://doi.org/10.1016/s0009-2797(02)00212-0

Verkman AS, Smith AJ, Phuan P, et al (2017) The aquaporin-4 water channel as a potential drug target in neurological disorders. Expert Opinion on Therapeutic Targets 21:1161-1170. https://doi.org/10.1080/14728222.2017.1398236

Vlassara H (2001) The AGE-receptor in the pathogenesis of diabetic complications. Diabetes/Metabolism Research and Reviews 17:436-443. https://doi.org/10.1002/dmrr.233

Wang C-Y, Liu H-J, Chen H-J, et al (2011) AGE-BSA down-regulates endothelial connexin43 gap junctions. BMC Cell Biol 12:19. https://doi.org/10.1186/1471-2121-12-19 
Wang H-L, Lai TW (2015) Optimization of Evans blue quantitation in limited rat tissue samples. Sci Rep 4:6588. https://doi.org/10.1038/srep06588

Wang X-J (2019) Elevated levels of $\alpha$-dicarbonyl compounds in the plasma of type II diabetics and their relevance with diabetic nephropathy. Journal of Chromatography B 7

Xing L, Yang T, Cui S, Chen G (2019) Connexin Hemichannels in Astrocytes: Role in CNS Disorders. Front Mol Neurosci 12:23. https://doi.org/10.3389/fnmol.2019.00023

Zanotto C, Simão F, Gasparin MS, et al (2017) Exendin-4 Reverses Biochemical and Functional Alterations in the BloodBrain and Blood-CSF Barriers in Diabetic Rats. Mol Neurobiol 54:2154-2166. https://doi.org/10.1007/s12035016-9798-1

Zhang FF, Morioka N, Kitamura T, et al (2015) Proinflammatory cytokines downregulate connexin 43 -gap junctions via the ubiquitin-proteasome system in rat spinal astrocytes. Biochemical and Biophysical Research Communications 464:1202-1208. https://doi.org/10.1016/j.bbrc.2015.07.105

Zhang M, An C, Gao Y, et al (2013) Emerging roles of Nrf2 and phase II antioxidant enzymes in neuroprotection. Progress in Neurobiology 100:30-47. https://doi.org/10.1016/j.pneurobio.2012.09.003

Zhao J, Liu L, Li X, et al (2019) Neuroprotective effects of an Nrf2 agonist on high glucose-induced damage in HT22 cells. Biol Res 52:53. https://doi.org/10.1186/s40659-019-0258-z

\section{Statements \& Declarations}

\section{Author Contributions}

C.A.G. and L.J.L. designed the study. L.J.L., K.M.W., L.R., L.D.B., E.B., V.G.D., performed laboratory experiments and collected data. L.J.L. and K.M.W performed statistical analyses. C.A.G., L.J.L, K.M.W., and L.R. wrote the manuscript. All authors edited and approved the manuscript.

\section{Data Availability}

The data used to support the findings of this study are available from the corresponding author upon request.

\section{Disclosure of Potential Conflicts of Interest}

The authors declare that they have no conflicts of interest.

\section{Funding}

This work was supported by the Conselho Nacional de Desenvolvimento Científico e Tecnológico (CNPq), Coordenação de Aperfeiçoamento de Pessoal de Nível Superior (CAPES), Fundação de Amparo à Pesquisa do Estado do Rio Grande do Sul (FAPERGS) and National Institute of Science and Technology for Excitotoxicity and Neuroprotection (INCTEN/CNPq).

\section{Ethics approval}

All animal experiments were carried out in accordance with the National Institute of Health Guide for the Care and Use of Laboratory Animals (NIH Publications No. 80-23), and all procedures were previously approved by the local Animal Care Ethical Committee (CEUA-UFRGS; project number 33663). All efforts were made to minimize animal suffering and reduce the number of animals used. 


\section{Figure legends}

Fig 1 Effect of ICV administration of MG on function and integrity of the BBB. All measurements were made seventytwo hours after surgery. The concentration of EBD was extracted from total brain and measured by spectroscopy (absorbance at 620nm) (A) and CSF/serum albumin ratio was measured by bromocresol green assay kit (B). Data expressed as means \pm S.E.M $(\mathrm{N}=4-6$ animals per group). Data were analyzed by Student's $t$-test assuming $\mathrm{p}<0.05$ and the statistical significance represents difference from the SHAM group

Fig 2 Effect of ICV administration of MG on the astroglial connectivity. All measurements were made seventy-two hours after surgery. Hippocampal slices of rats were dissected out and immunocontents of hippocampal Aquaporin 4 (AQP4) (A) and Conexin 43 (Cx43) (B) were determined by western blotting and normalized by actin, assuming SHAM value as $100 \%$. Data expressed as means \pm S.E.M $(\mathrm{N}=3-11$ animals per group $)$ and analyzed by Student's $t$-test assuming $\mathrm{p}<0.05$ and the statistical significance represents difference from the SHAM group

Fig 3 Effect of ICV administration of the RAGE signaling. All measurements were made seventy-two hours after surgery. Hippocampal slices of rats were dissected out and the RAGE content were determined by western blotting and normalized by actin (A), and S100B, measured by ELISA (B); S100B and HMGB1 (C and D, respectively) mRNA expression were measured. Data expressed as means \pm S.E.M (N = 5- 13 animals per group) assuming SHAM as $100 \%$. SHAM value of $\mathrm{S} 100 \mathrm{~B}$ content was $0.67 \mathrm{nmol} / \mathrm{mg}$ protein in hippocampus. Data were analyzed by Student's $t$-test, assuming $\mathrm{p}<0.05$ and the statistical significance represents difference from the SHAM group

Fig 4 Effect of ICV administration of MG on the Nrf2/HO-1 pathway. All measurements were made seventy-two hours after surgery. Hippocampal slices of rats were dissected out and mRNA expression of NfkB (A), Nrf2 (B) and HO-1 (C) were measured. Data expressed as means \pm S.E.M ( $N=5-8$ animals per group) assuming SHAM value as $100 \%$ and analyzed by Student's $t$-test, assuming $\mathrm{p}<0.05$ and the statistical significance represents difference from the SHAM group 
A

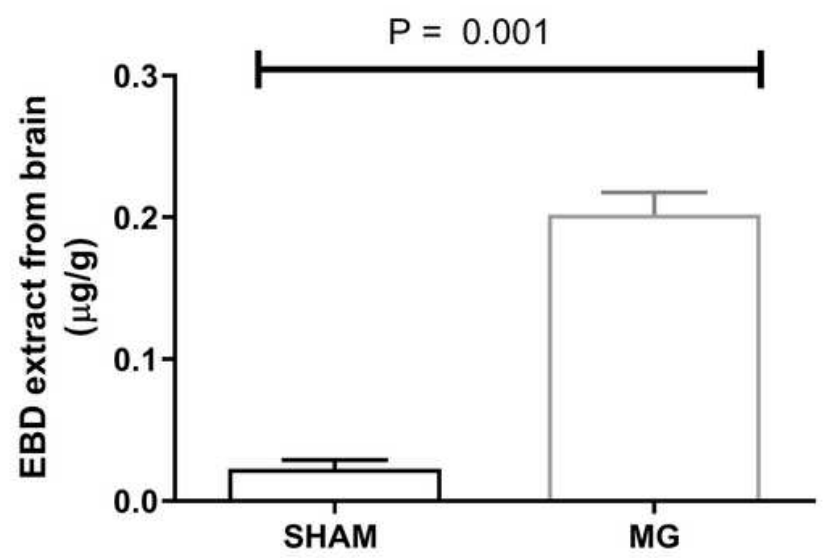

B

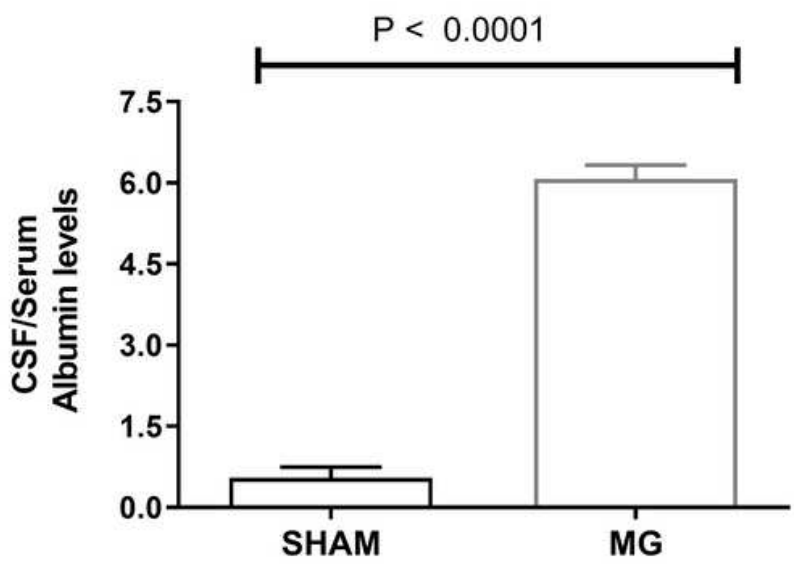

Figure 1

Effect of ICV administration of MG on function and integrity of the BBB. All measurements were made seventy two hours after surgery. The concentration of EBD was extracted from total brain and measured by spectroscopy (absorbance at $620 \mathrm{~nm}$ ) (A) and CSF/serum albumin ratio was measured by bromocresol green assay kit (B). Data expressed as means \pm S.E.M $(\mathrm{N}=4-6$ animals per group). Data were analyzed by Student's t-test assuming $\mathrm{p} \otimes 0.05$ and the statistical significance represents difference from the SHAM group

A

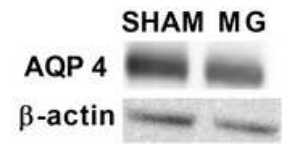

$35 \mathrm{kDa}$

42 kDa

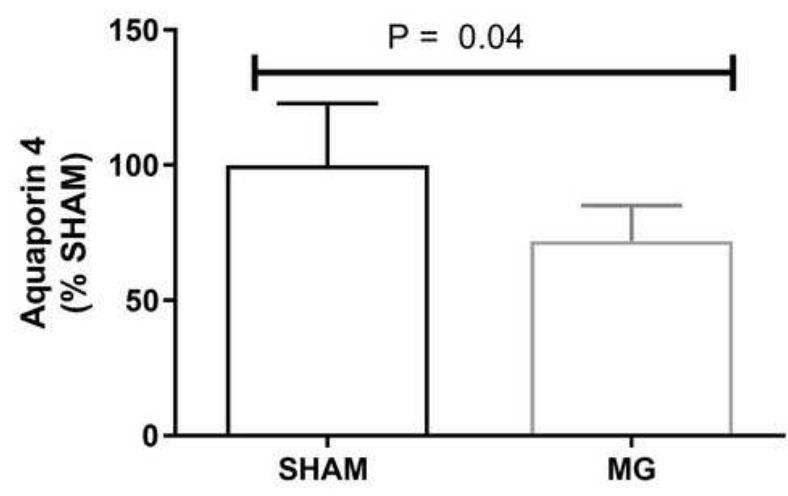

B
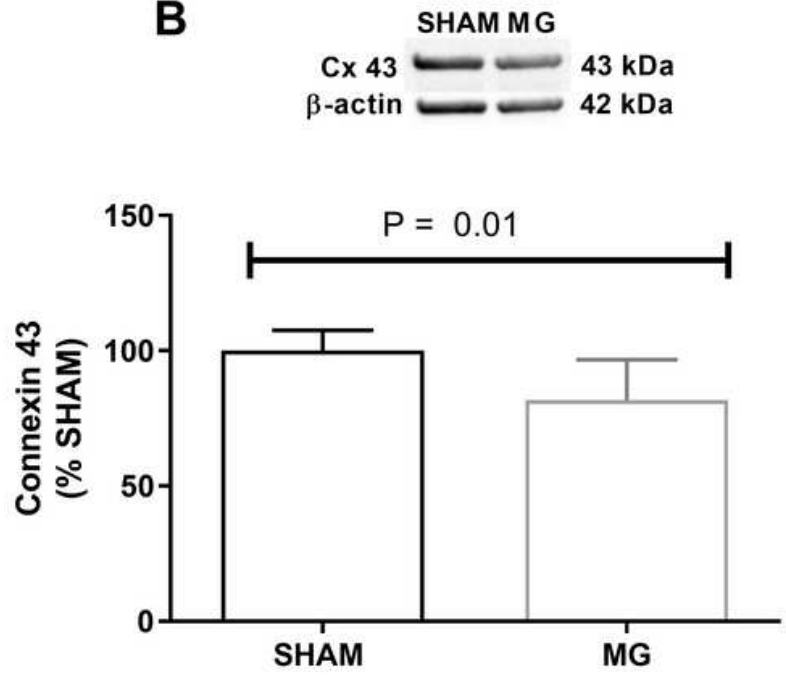

Figure 2 
Effect of ICV administration of MG on the astroglial connectivity. All measurements were made seventytwo hours after surgery. Hippocampal slices of rats were dissected out and immunocontents of hippocampal Aquaporin 4 (AQP4) (A) and Conexin 43 (Cx43) (B) were determined by western blotting and normalized by actin, assuming SHAM value as $100 \%$. Data expressed as means \pm S.E.M $(\mathrm{N}=3-11$ animals per group) and analyzed by Student's t-test assuming $\mathrm{p} \otimes 0.05$ and the statistical significance represents difference from the SHAM group
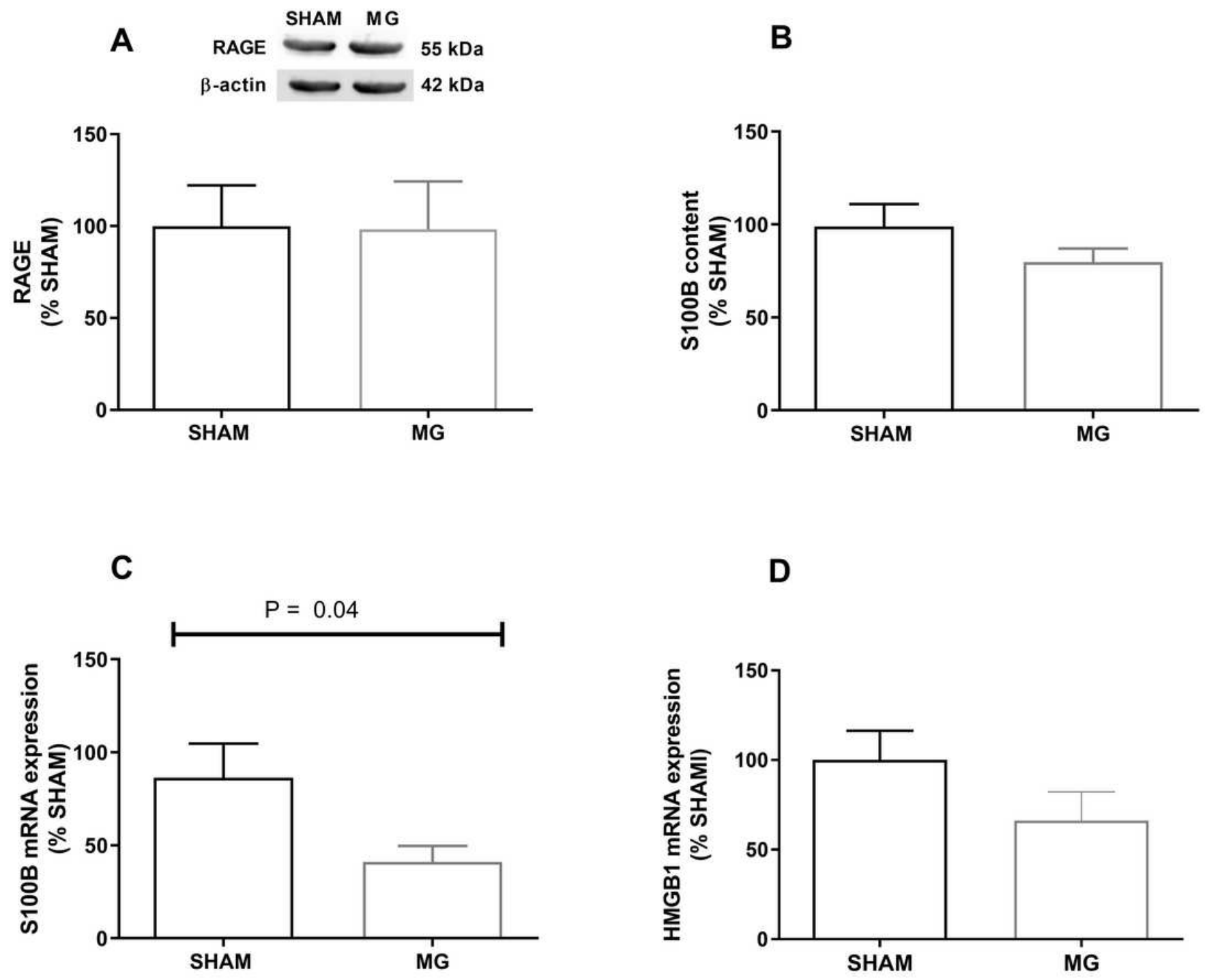

Figure 3

Effect of ICV administration of the RAGE signaling. All measurements were made seventy-two hours after surgery. Hippocampal slices of rats were dissected out and the RAGE content were determined by western blotting and normalized by actin (A), and S100B, measured by ELISA (B); S100B and HMGB1 (C and D, respectively) mRNA expression were measured. Data expressed as means \pm S.E.M $(N=5-13$ animals per group) assuming SHAM as $100 \%$. SHAM value of S100B content was $0.67 \mathrm{nmol} / \mathrm{mg}$ protein in 
hippocampus. Data were analyzed by Student's t-test, assuming $\mathrm{p} \otimes 0.05$ and the statistical significance represents difference from the SHAM group

A

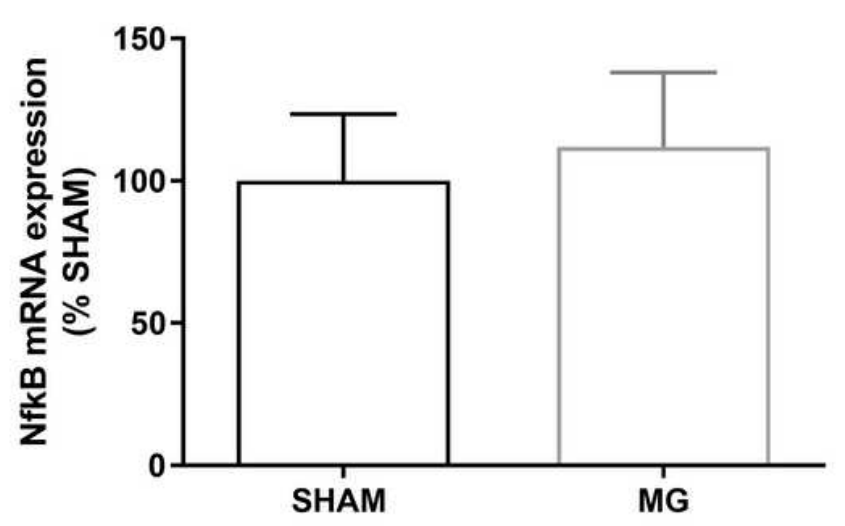

B

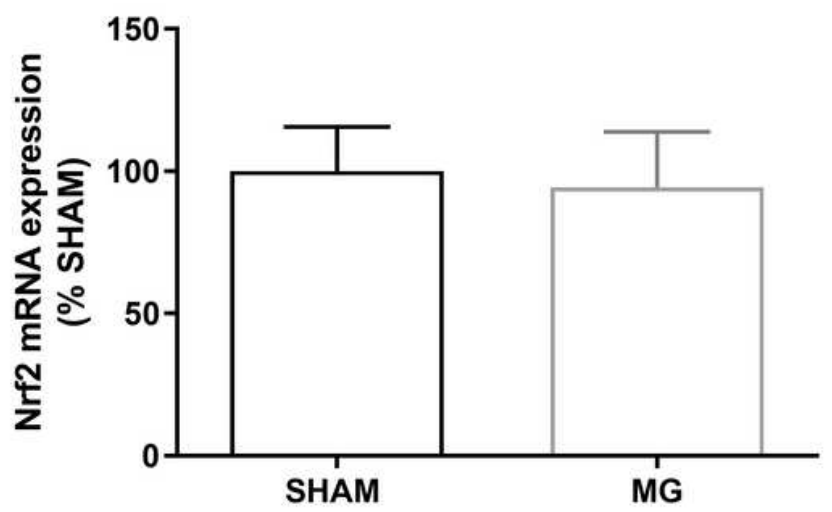

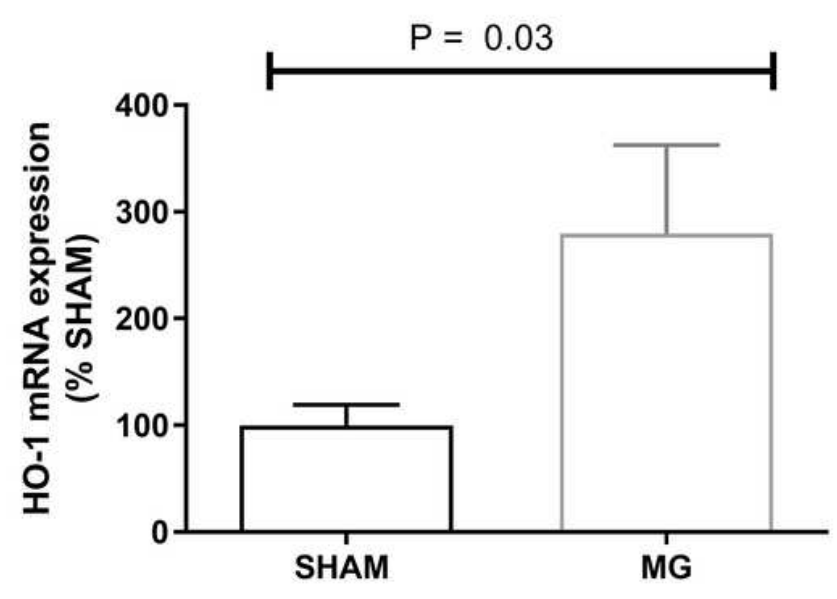

Figure 4

Effect of ICV administration of MG on the Nrf2/HO-1 pathway. All measurements were made seventy-two hours after surgery. Hippocampal slices of rats were dissected out and mRNA expression of NfkB (A), Nrf2 (B) and HO-1 (C) were measured. Data expressed as means \pm S.E.M ( $N=5-8$ animals per group) assuming SHAM value as $100 \%$ and analyzed by Student's t-test, assuming $\mathrm{p} \otimes 0.05$ and the statistical significance represents difference from the SHAM group 